\title{
Prediction of a common neutralizing epitope of H5N1 avian influenza virus by in silico molecular docking
}

\author{
YAN YuanQing, LI ShaoWei ${ }^{\dagger}$, YANG ChunYan, LUO WenXin, WANG MingQiao, CHEN YiXin, \\ LUO HaiFeng, WU Ting, ZHANG Jun \& XIA NingShao
}

\begin{abstract}
National Institute of Diagnostics and Vaccine Development in Infectious Diseases/Key Laboratory of the Ministry of Education for Cell Biology and Tumor Cell Engineering, Xiamen University, Xiamen 361005, China
\end{abstract}

The H5N1 avian influenza virus (AIV) has widely spread in Asia, Europe and Africa, making a large amount of economic loss. Recently, our research group has screened a common neutralizing monoclonal antibody named $8 \mathrm{H} 5$, which can neutralize almost all H5 subtype AIV ever isolated so far. Obviously, this monoclonal antibody would benefit for research and development of the universal AIV vaccine and design of the drug against H5N1 AIV in high mutation rate. In this study, the homology modeling was applied to generate the 3D structure of $8 \mathrm{H} 5$ Fab fragment, and "canonical structure" method was used to define the specified loop conformation of CDR regions. The model was subjected to energy minimization in cvff force field with Discovery module in Insight II program. The resulting model has correct stereochemistry as gauged from the Ramachandran plot calculation and good 3D-structure compatibility as assessed by interaction energy analysis, solvent accessible surface (SAS) analysis, and Profiles-3D approach. Furthermore, the 8H5 Fab model was subjected to docking with three $\mathrm{H} 5$ subtype hemagglutinin (HA) structures deposited in PDB (ID No: 1jsm, 2ibx and 2fk0) respectively. The result indicates that the three docked complexes share a common binding interface, but differ in binding angle related with HA structure similarity between viral subtypes. In the light of the three HA interfaces with structural homology analysis, the common neutralizing epitope on HA recognized by $8 \mathrm{H} 5$ consists of 9 incontinuous amino acid residues: $\mathrm{Asp}^{68}, \mathrm{Asn}^{72}, \mathrm{Glu}^{112}, \mathrm{Lys}^{113}, \mathrm{Ile}^{114}$, $\mathrm{Pro}^{118}, \mathrm{Ser}^{120}, \mathrm{Tyr}^{137}$, $\mathrm{Tyr}^{252}$ (numbered as for $1 \mathrm{jsm}$ sequence). The primary purpose of the present work is to provide some insight into structure and binding details of a common neutralizing epitope of H5N1 AIV, thereby aiding in the structure-based design of universal AIV vaccines and anti-virus therapeutic drugs.

H5N1 avian influenza virus, molecular docking, hemagglutinin, neutralizing epitope

Since late 2003, H5N1 avian influenza virus (AIV) has widely spread all over the world, not only resulting in mass poultry death, but also leading to high mortality in human patients. Hemagglutinin (HA), the major surface protein of AIV, not only mediates viral attachment and entry into the host cell by binding to sialic acid receptors, but also is responsible for protective humoral immunity by neutralizing antibody. However, the high mutation ratio of HA makes it very difficult to control AIV. Recently we have constructed an antibody panel that contained 388 antibodies possessing $\mathrm{H} 5$ hemagglutinin inhibited (HI) activity. In this panel, one antibody
$8 \mathrm{H} 5$ can strongly neutralize all of the 46 viruses isolated from different clades, locations and hosts ${ }^{[1]}$. The research on the structure of this common neutralizing antibody may contribute to a breakthrough in seeking methods to overcome the high mutation of H5N1.

\footnotetext{
Received May 15, 2007; accepted December 26, 2007 doi: 10.1007/s11434-008-0161-4

'Corresponding author (email: shaowei@xmu.edu.cn)

Supported by the National Natural Science Foundation of China (Grant Nos 30500092, 30640017 and 30600106), National Science and Technology Project in the 10th Five-Year Period (Grant No. 2004BA519A73), Key Projects in the National Science \& Technology Pillar Program (Grant No. 2006BAI01B06), Major Science and Technology Project of Fujian Province (Grant No. 2004YZ01), Key Science and Technology Project of Fujian Province (Grant No. 2005Y020)
} 
Because antibody has an intrinsic nature with conserved amino acid sequence and typical configuration in 3D structure, the structure can be modeled by some structural templates from Brookhaven PDB where a large number of X-ray resolved antibody structures deposit ${ }^{[2,3]}$. The homology modeling method has been widely used to search for the epitopes of antigen and to improve the binding affinity. Frequently 5 of the 6 complementarity-determining regions (CDRs) (all except H3) of antibody fall into one of between 2 and 6 structural classes, referred to as canonical classes ${ }^{[3,4]}$. Based on the determinants of the canonical structures, the most accurate method for building the model of antibody can be developed ${ }^{[5]}$. Here we will describe the structure modeling of $8 \mathrm{H} 5$ using the canonical structure method and the docking pattern between $8 \mathrm{H} 5$ and three HAs deposited in PDB. The docking results can shed light on the common neutralizing epitope of $\mathrm{H} 5$, thus providing invaluable information for the development of the potential vaccine, design of antiviral drug and research on the broad-spectrum therapeutic antibody.

\section{Materials and methods}

\subsection{Antibody sequence preparation and antigen structure refinement}

The monoclonal antibody $8 \mathrm{H} 5$ was raised in our lab, and the gene of variable regions was cloned from the hybrid plasmoma cell and verified with sequencing by BoYa Co. in Shanghai ${ }^{[1]}$. The primary amino acid sequence of the variable regions is listed below: Variable region of light chain, EIVLTQSPAIMSASLGEKVTMSCRASSSVNFVYWYQQRSDASPKLLIYYSSNLAPGVPPRFSGSGSGNSYSLTISGLEGEDAATYYCQHFTSSPYTFGGGTKLEIKRLE; Variable region of heavy chain, QVQLQQSGAELMKPGASVKISCKATGYTFSNYWIEWIKQRPGHGLEWIGEILPGSDRTNYNGKFKGKATFTADTSSNTAHMQLSSLTSEDSAVYYCANRYDGYYFGLDYWGQGTSVTVSS.

There were $3 \mathrm{X}$-ray resolved HA antigen structures depositing in PDB. The PDB IDs were as follows: $1 \mathrm{jsm}$ (Virus strain: A/DUCK/SINGAPORE/3/97, Host: Bird), 2fk0 (Virus strain: A/VIETNAM/1203/2004, Host: Human) and 2bix (Virus strain: VN1194, Host: Human) ${ }^{[6-8]}$. The structure of $1 \mathrm{jsm}$ was refined in Insight II package (Accelrys Company) before docking with the antibody. Because the other 2 structures were deposited as the trimers, the monomers were unmerged and then sub- jected to refinement before docking.

\subsection{Chothia numbering of antibody sequence and canonical structure analysis}

According to Chothia numbering scheme, the amino acid insertion and deletion site in antibody were located in residues L31, L95, L106, H31, H52, H82 and H100 ${ }^{[2]}$. For the variable domain in light chain (VL), the conserved residue cysteine was always located in residues 23 and 88 which were 1 residue ahead of the start point of CDR-L1 and CDR-L3 respectively. The residue after CDR-L1, which ranged from residue 24 to 33 , was always Trp and the residues after CDR-L3, which ranged from residue 89 to 97 , were always Phe-Gly. CDR-L2, which ranged from residue 50 to 56 , always started sixteen residues after the end of L1. The residues before CDR-L2 generally were Ile-Tyr. For the variable domain in heavy chain $(\mathrm{VH})$, the conserved residue cysteine was always located in residues 22 and 92, which were 4 and 3 residues ahead of the start point of CDR-H1 and CDR$\mathrm{H} 3$, respectively. The residue after CDR-H1, which ranged from residue 26 to 32, was always Trp and the residues after CDR-H3, which ranged from residue 95 to 102, were always Trp-Gly. CDR-H2, which ranged from residue 52 to 56 , always started 15 th residues after the end of $\mathrm{H} 1$. The residues before CDR-H2 typically were Leu-Glu-Trp-Ile-Gly and the residues after CDR-H2 were Lys/Arg-Leu/Ile/Val/Phe/Thr/Ala-Thr/Ser/Ile/Ala. Inspite of the high sequence variability in CDRs, five of them (all except CDR-H3) can assume just a small repertoire of main-chain conformation, called canonical structures, and each of the 5 CDRs fell into 1 of between 2 and 6 canonical structure classes. The conformations for canonical structures were determined by the length of the loops and by the presence of key residues at specific positions in the antibody sequence (either within the loops or in the framework regions) ${ }^{[5]}$.

\subsection{Molecular modeling of $L$ and $H$ chains}

In the Homology module of Insight II software package, $\mathrm{L}$ and $\mathrm{H}$ sequences were aligned with the sequences from PDB using FASTA method ${ }^{[9,10]}$. The protein structures with $>50 \%$ homology to the targets were selected as templates. The conserved residue coordinates in framework were first assigned. For the canonical structures, the coordinates from PDB with the same canonical classes as targets were adopted and assigned to the $\mathrm{L}$ or $\mathrm{H}$ sequences. For the $\mathrm{CDR}-\mathrm{H} 3$, the sequence was also sent to PDB to seek the best loop coordinate, and the 
one with least RMSD was selected as the template. The terminals and the splice region of the structures were repaired by the Refine program in Homology module in Insight II package. The structures were released with the RMSD less than $0.5 \AA$ before proceeding to the next step. And then the models were subjected to optimization in the consistent-valence force field using the conjugate gradient method, the steepest descent method and the molecule dynamics method in the Discover module.

\subsection{Structural pairing of $L$ and $H$ chains}

The residues at the L-H interface were highly conserved among almost all antibodies, which were: L33-L39, L43-L47, L84-L90, L98-L104, H34-H40, H44$\mathrm{H} 48, \mathrm{H} 88-\mathrm{H} 94, \mathrm{H} 103-\mathrm{H} 109^{[3,11]}$. In order to obtain the fab fragment, the $\mathrm{L}$ and $\mathrm{H}$ domains were packed together by a least-squares fit of the main-chain atoms of residues in the interface. Only the outcome with RMSD less than $0.5 \AA$ was adopted. The structures of $\mathrm{L}$ and $\mathrm{H}$ were merged to an assembly named $8 \mathrm{H} 5 \mathrm{Fab}$. In program BUMP, the residues in serious collision were subjected to torsion revision. After that, the backbone atoms of $8 \mathrm{H} 5 \mathrm{Fab}$ were fixed, and 1000 steps of optimization in the consistent-valence force field were carried out by the conjugate gradient method and the steepest descent method. The energy minimized $8 \mathrm{H} 5 \mathrm{Fab}$ was tested by residue compatibility with Profile-3D program, solvent accessible surface and interaction energy analysis.

\subsection{Molecular docking of 8H5Fab and HAs}

The $8 \mathrm{H} 5 \mathrm{Fab}$ and the optimized antigens were subjected to Zdock in Zdockpro module in Insight II package ${ }^{[12]}$. The $8 \mathrm{H} 5 \mathrm{Fab}$ was taken as receptor and the antigens were ligand. In order to save the computing time, the regions of HA2 and the constant regions of $8 \mathrm{H} 5 \mathrm{Fab}$ were blocked. From the Zdock output file, the first 30 poses were selected for further Rdock analysis ${ }^{[13]}$. From the Zdock scores, Rdock scores and the CDR binding regions, the complexes were screened for further study.

\subsection{Solvent accessible surface analysis of complexes and epitope candidates prediction}

Based on the complexes and the individual HAs, $8 \mathrm{H} 5 \mathrm{Fab}$, the values of solvent accessible surface (SAS) area were calculated for each residue using a probe radius of $1.4 \AA$. The residues of HAs with more than $7 \AA^{2}$ decrease in SAS during binding were regarded as the epitope candidates ${ }^{[14-16]}$. Moreover, an HA residue was considered to belong to the epitope if it was significantly buried in the complex and at least one atom of the residue made an atomic contact with an atom of the antibody fragment, at a distance compatible with either a hydrogen bond, a salt bridge or a Van der Waals interaction.

\section{Results}

\subsection{Molecular modeling of 8H5Fab}

(i) The templates for $8 \mathrm{H} 5 \mathrm{Fab}$ modeling and its "canonical structure" classes. In the light of the homologous similarity, the templates for the $\mathrm{V}_{\mathrm{L}}$ or $\mathrm{V}_{\mathrm{H}}$ were screened from the PDB. From the multiple sequence alignment between the target sequences and the templates, the conserved residues, the conserved frameworks and CDRs can be identified (Figure 1).

The sequences were labeled with the Chothia numbering method (Table 1). For CDR-L1, there was one amino acid residue deletion located in residue 31 . One and three residues insertion occurred in CDR-H2 (P52a) and CDR-H3 (F100a, G100b and L100c), respectively. Based on the length of the loops and the presence of key residues at specific positions in the antibody sequence, the canonical structure classes were defined (Table 1). Three of them (CDR-L1, CDR-L3 and CDR-H1) belonged to class 1 and the other two were to class $2^{[5]}$.

(ii) Structure of the $8 \mathrm{H} 5 \mathrm{Fab}$. The modeled structure of the $8 \mathrm{H} 5 \mathrm{Fab}$ is shown in Figure 2, its chains $\mathrm{L}$ and chain $\mathrm{H}$ having the common features of a typical antibody. Chain L contained 211 aa totally with 109 residues located in variable domain and the rest 102 in constant domain. Chain H contained 219 aa with 120 residues were located in variable domain and the rest 99 in constant domain. Each domain was packed with two face-to-face $\beta$ sheets, linked together by a conserved disulfide bridge and inter-chain loops. The disulfide bonds were located at $\mathrm{Cys}^{23}-\mathrm{Cys}^{88}$ in VL and at $\mathrm{Cys}^{22}$ $\mathrm{ys}^{92}$ in VH. Also there were two pairs of disulfide bonds in the constant domains. Like other antibodies, the average bond-length of disulfide bonds was about $2.0 \AA$ which was the standard length to stabilize the molecule structure.

(iii) The verification of the $8 \mathrm{H} 5 \mathrm{Fab}$ structure.

(1) Energy analysis. As shown in Table 2, the total energy of model $\mathrm{H}$ and model $\mathrm{L}$ was 3637.96 and $4551.28 \mathrm{kal}$, respectively. However the energy of $8 \mathrm{H} 5 \mathrm{Fab}$ was $7875.71 \mathrm{kcal}$, which was $313.53 \mathrm{kal}$ less 

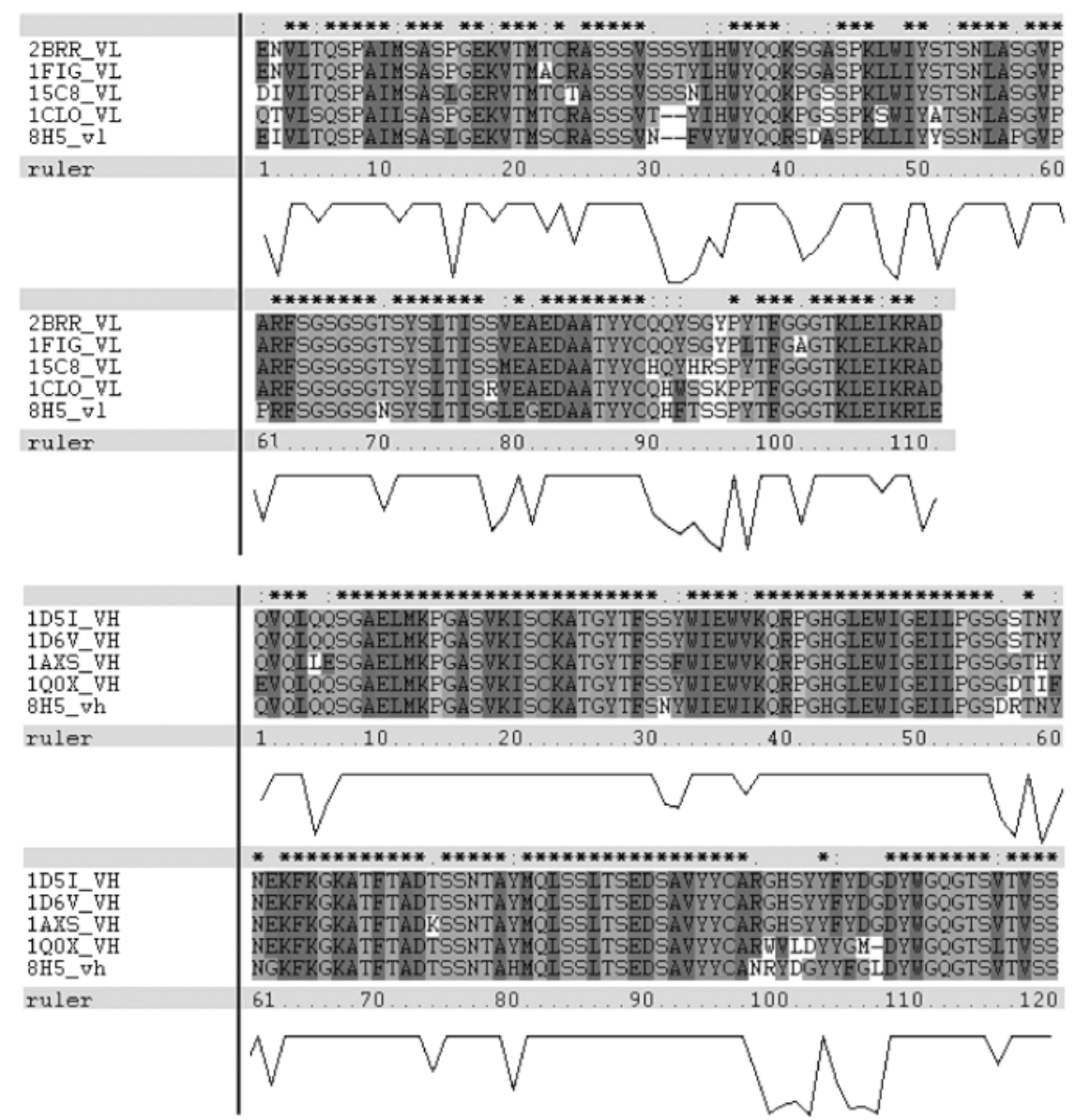

Figure 1 Sequence alignment between the $\mathrm{V}_{\mathrm{L}}, \mathrm{V}_{\mathrm{H}}$ and the templates (clustal $\mathrm{X}$ ).

Table 1 The sequence numbering and canonical structure classification

\begin{tabular}{llclc}
\hline & \multicolumn{1}{c}{ Amino acid } & Original numbering & \multicolumn{1}{c}{ Chothia numbering } & Canonical structure \\
\hline CDR-L1 & RASSSVNFVY & $24-33$ & 24252627282930323334 & 1 \\
CDR-L2 & YSSNLAP & $49-55$ & 50515253545556 & 2 \\
CDR-L3 & QHFTSSPYT & $88-96$ & 899091929394959697 & 1 \\
CDR-H1 & GYTFSNY & $26-32$ & 26272829303132 & 1 \\
CDR-H2 & LPGSDR & $52-57$ & $5252 \mathrm{a} 53545556$ & 2 \\
CDR-H3 & RYDGYYFGLDY & $99-109$ & $95-100100 \mathrm{a} 100 \mathrm{~b} 100 \mathrm{c} 101102$ & \\
Others & LSSLT & $83-87$ & $8282 \mathrm{a} 82 \mathrm{~b} 82 \mathrm{c} 83$ & \\
\hline
\end{tabular}

than the total energy of the $\mathrm{L}$ and $\mathrm{H}$ models. The loss of energy indicated that the $8 \mathrm{H} 5 \mathrm{Fab}$ became more stable after L-H pairing. Moreover, as shown in Table 3, the loss of energy which was almost the same as interacting energy between model $\mathrm{H}$ and model $\mathrm{L}$ implied that the two models had a tendency to form a dimmer, and the pairing was driven by the free energy. This obeys the second law of thermodynamics.

(2) SAS analysis. With $1.4 \AA$ solvent radius, the SAS for model L and $\mathrm{H}$ was 11580.41 and $11852.29 \AA^{2}$, respectively. However the SAS for $8 \mathrm{H} 5 \mathrm{Fab}$ was $1973709 \AA^{2}$, about $3600 \AA^{2}$ less than the total SAS of model L and H. For the typical antibody structure, Cyrus Chothia reported that the loss SAS between $\mathrm{V}_{\mathrm{H}}$ and $\mathrm{V}_{\mathrm{L}}$ domains was about $1800 \AA^{2}$ after pairing ${ }^{[11]}$. $8 \mathrm{H} 5 \mathrm{Fab}$ consisted of $\mathrm{V}_{\mathrm{H}}-\mathrm{V}_{\mathrm{L}}$ and $\mathrm{C}_{\mathrm{H}}-\mathrm{C}_{\mathrm{L}}$, and the buried surface between model $\mathrm{L}$ and $\mathrm{H}$ was twice that of the typical $1800 \AA^{2}$ between two partner domains, which further verified the model $8 \mathrm{H} 5 \mathrm{Fab}$.

(3) Hydrogen bond analysis. At the interface of the model $\mathrm{L}$ and $\mathrm{H}$, there were 13 pairs of hydrogen bonds (Table 4). Seven of them were located in the variable regions and the other 6 in the constant regions. The hydrogen bonds were one of the main forces between the 


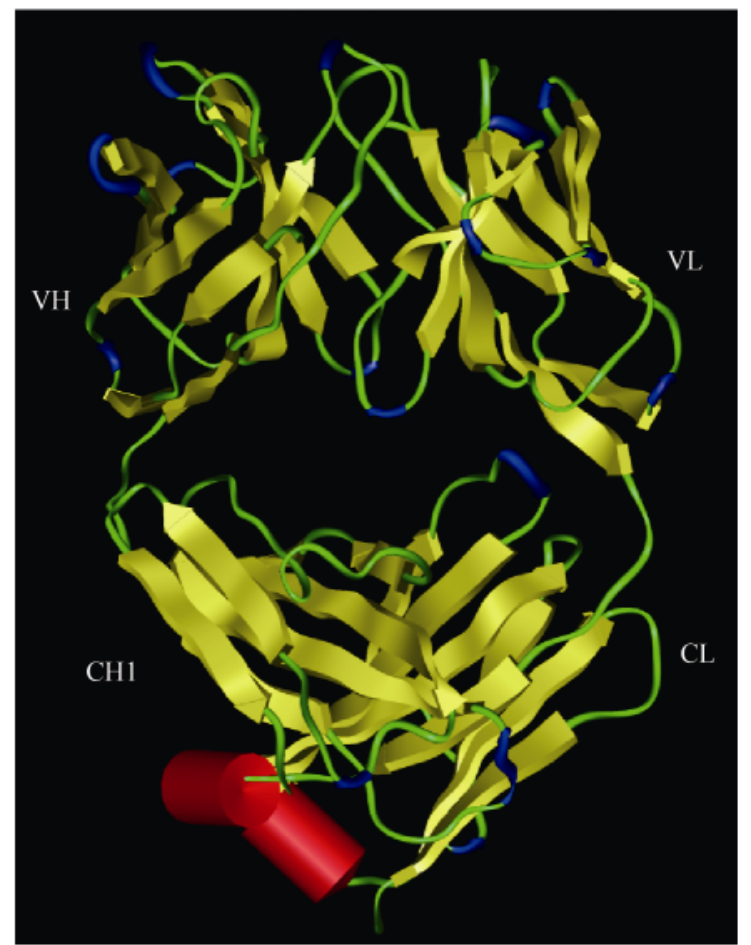

Figure 2 The 3D structure of the model 8H5Fab.

interacting domains, playing an important role in the stability of the model $8 \mathrm{H} 5 \mathrm{Fab}$.

(4) Ramachandran plot. In Figure 3, the ramachandran plot of the 6 loops showed that $80 \%$ residues of the CDRs were in the most favored regions, meaning that the model $8 \mathrm{H} 5 \mathrm{Fab}$ was in good 3D-tructure compatibility. The $8 \mathrm{H} 5 \mathrm{Fab}$ model superimposed with crystallographically-determined antibody structures showed good overall agreement exception to the loops.

(5) Profile-3D analysis. When the $8 \mathrm{H} 5 \mathrm{Fab}$ was subjected to profile-3D test in Insight II package, the overall self-compatibility score for $8 \mathrm{H} 5 \mathrm{Fab}$ was 216.91 , while the score expected for a valid protein of this size was 196.23 and for a protein of this size, a score of 88.30 or less would indicate a structure that was almost certainly incorrect. Moreover, the test scores of all amino acid residues were above zero (Figure 4). All of this indicated that the model $8 \mathrm{H} 5 \mathrm{Fab}$ was in a very good and reasonable conformation.

\subsection{The molecular docking between $8 \mathrm{H} 5 \mathrm{Fab}$ and the HAs}

In Figure 5, the binding sites of 8H5 in HAs were quite near the receptor binding sub-domains ${ }^{[8]}$, suggesting that the antibody $8 \mathrm{H} 5$ might compete for the binding sites with the receptor and block the avian influenza virus infection, i.e. the so-called neutralizing activity.

On keeping the $8 \mathrm{H} 5 \mathrm{Fab}$ location still, and superimposing the $38 \mathrm{H} 5 \mathrm{Fab}-\mathrm{HAs}$ in facility for the analysis, it was clear that $8 \mathrm{H} 5 \mathrm{Fab}$ bound to $1 \mathrm{jsm}$ and 2 bix HAs almost in the same way, but for the $2 \mathrm{fk} 0 \mathrm{HA}$, there was some deviation, and the binding sites were very close to the His/Lys patch. In detail, it showed that the side chains of $8 \mathrm{H} 5 \mathrm{Fab}$ for the binding were differently involved (Figure 5(b)) just as the binding angles' difference (Figure 5(a)). Moreover, it was reported that $1 \mathrm{jsm}$ was from bird and 2ibx was from human, which indicated that the preference binging of $8 \mathrm{H} 5$ was unrelated to the hosts. Comparing $1 \mathrm{jsm}$ and $2 \mathrm{ibx}$, we found that the HA structure from $2 \mathrm{fk} 0$ was more similar to that from $\mathrm{H} 1$, which implied that $2 \mathrm{fk} 0$ was the transition

Table 2 The energy analysis of model L, model $\mathrm{H}$ and model 8H5Fab

\begin{tabular}{|c|c|c|c|}
\hline & Model L (kcal) & Model H (kcal) & 8H5Fab (kcal) \\
\hline Van der Waals energy & 1903.96936 & 1830.8492 & 3506.664 \\
\hline Repulsive energy & 9630.30371 & 9506.7725 & 19408.84 \\
\hline Dispersive energy & -7726.33447 & -7675.923 & -15902.18 \\
\hline Coulomb energy & 1.61702 & 789.50665 & 705.7458 \\
\hline Bond energy & 536.83484 & 457.26556 & 994.1004 \\
\hline Theta energy & 874.28912 & 1071.5287 & 1945.818 \\
\hline Phi energy & 251.47975 & 270.37045 & 521.8502 \\
\hline Out of plane energy & 7.66596 & 15.04769 & 22.71366 \\
\hline Bond-bond energy & 15.56962 & 33.08727 & 48.65688 \\
\hline Bond-theta energy & 44.38714 & 81.77821 & 126.1654 \\
\hline Theta-theta energy & 21.44454 & 31.37887 & 52.82341 \\
\hline Theta-theta-phi energy & -19.29458 & -29.53335 & -48.82793 \\
\hline Bond-bond(1-3) energy & 0 & 0 & 0 \\
\hline Op-op energy & 0 & 0 & 0 \\
\hline Phi-phi energy & 0 & 0 & 0 \\
\hline Hydrogen-bond energy & 0 & 0 & 0 \\
\hline Total energy & 3637.96289 & 4551.2778 & 7875.707 \\
\hline
\end{tabular}


Table 3 The interaction energy of model $\mathrm{L}$ and model $\mathrm{H}$

\begin{tabular}{l}
\hline Summary for objects: $\mathrm{L}$ and $\mathrm{H}$ \\
\hline Van der Waals energy $=-225.68803 \mathrm{kcal}$ \\
Repulsion energy $=271.72363 \mathrm{kcal}$ \\
Dispersion energy $=-495.94510 \mathrm{kcal}$ \\
Coulomb energy $=-85.37601 \mathrm{kcal}$ \\
Total energy $=-311.06405 \mathrm{kcal}$ \\
\hline
\end{tabular}

Table 4 Hydrogen bonds formed between models $\mathrm{L}$ and $\mathrm{H}$ in $8 \mathrm{H} 5 \mathrm{Fab}$

\begin{tabular}{llll}
\hline H receptor & H donor & Distance $(\AA)$ & Angle $\left(^{\circ}\right)$ \\
\hline L:38:HE22 & H:39:OE1 & 2.05 & 155.28 \\
L:1:HE2 & H:46:OE1 & 1.83 & 156.42 \\
L:94:HG & H:50:OE2 & 2.18 & 153.78 \\
H:60:HD22 & L:1:OE1 & 2.41 & 145.53 \\
H:100B:HN & L:34:OH & 1.63 & 152.72 \\
L:36:HH & H:100C:O & 1.93 & 172.15 \\
L:43:HG & H:104:O & 1.93 & 156.76 \\
L:174:HG & H:164:ND1 & 2.43 & 148.73 \\
L:174:HG & H:164:NE2 & 2.30 & 151.36 \\
H:167:N & L:162:OG & 2.90 & NA \\
L:137:HD22 & H:180:OG & 2.34 & 165.49 \\
L:122:HN & H:212:O & 1.98 & 160.82 \\
L:122:HN & H:212:OXT & 2.38 & 130.38 \\
\hline
\end{tabular}

a) NA, No data was detected.

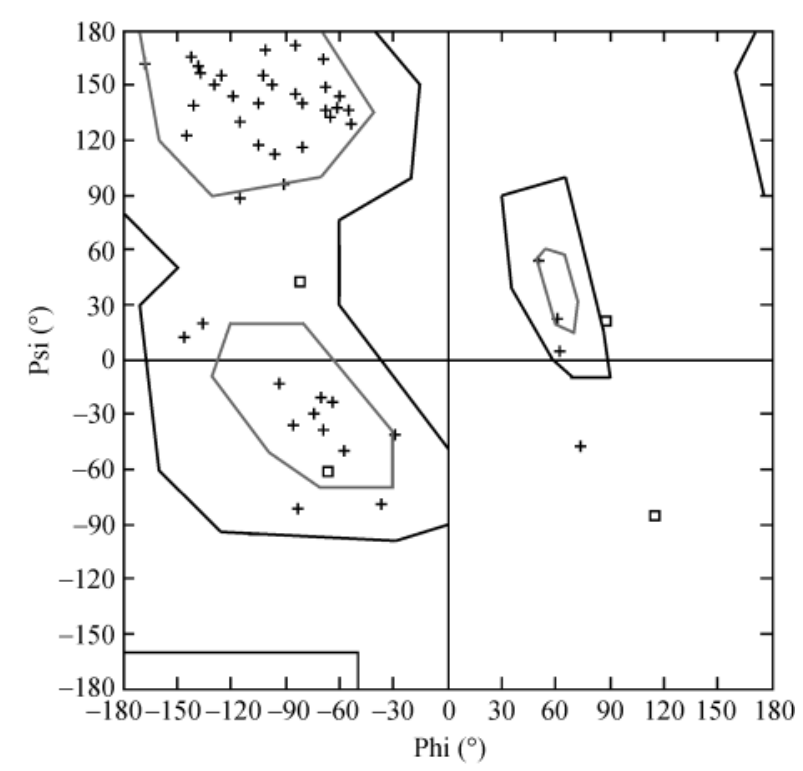

Figure 3 Ramachandran plot of CDRs.

structure from $\mathrm{H} 5$ to $\mathrm{H}^{[6]}$.

\subsection{The prediction of $\mathrm{HA}$ epitope}

(i) $\triangle \mathrm{SAS}$ analysis. The solvent accessible surface area of each residue in HA structures was calculated as the monomer and complex and the $\triangle \mathrm{SAS}$ was developed (Table 5). Some residues, such as $\mathrm{Phe}^{79}$ in $2 \mathrm{fk} 0, \mathrm{Ser}^{120}$ in $1 \mathrm{jsm}$ and $\mathrm{Ser}^{124}$ in 2 bix, were deeply buried in the complexes with a fraction larger than $90 \%$. Based on the complexes, the contacting residues within $4 \AA$ were also

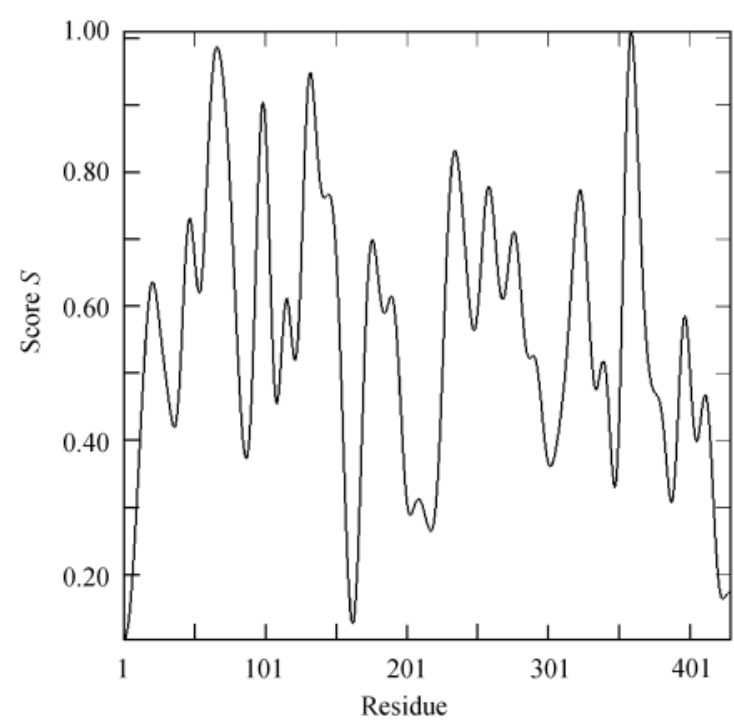

Figure 4 Profile-3D test of model 8H5Fab.

identified. Taking consideration of the $\triangle \mathrm{SAS}$ and the contacting residues, the epitopes were defined as follows (Table 5).

(ii) Sequence alignment analysis. From the experiment we carried out, we found that $8 \mathrm{H} 5$ could react with almost all of the $\mathrm{H} 5$ influenza viruses, indicating that the binding sites in HA were the common neutralizing epitope. Therefore, in the light of the sequence alignment (Figure 6) and the epitopes in 3 HAs shown in Table 5, the common $8 \mathrm{H} 5$ epitope consisted of (aa in 1jsm) Asp ${ }^{68}$, $\mathrm{Asn}^{72}, \mathrm{Glu}^{112}, \mathrm{Lys}^{113}, \mathrm{Ile}^{114}, \mathrm{Pro}^{118}, \mathrm{Ser}^{120}, \mathrm{Tyr}^{137}, \mathrm{Tyr}^{252}$.

(iii) Natural mutation analysis. In order to test the conservation of the common epitope in HAs, natural mutation analysis was performed. By blast on-line, 1000 H5 HA sequences from different virus isolates were screened and subjected to conservation analysis. As Table 6 shows, the amino acids were highly conserved with a ratio as high as $99 \%$, except for the $\operatorname{Ser}^{120}$ with $95.9 \%$ and $\mathrm{Tyr}^{252}$ with $87.2 \%$. The data also showed that the prediction of the common neutralizing epitope was convincing.

(iv) Hydrogen bond analysis in the epitope. There were many pairs of hydrogen bonds at the interface of HA-8H5Fab complex (Table 7). Most interestingly, the amino acid Lys $^{113}$ in 1 jsm $\left(\right.$ Lys $^{117}$ in $2 \mathrm{ibx}$ and Lys ${ }^{120}$ in $2 \mathrm{fk} 0$ ) contributed to the hydrogen bond formation in all the three HAs, indicating the most important role it played in the predicted common epitope. However, the amino acid Lys worked as the proton donor in $2 \mathrm{fk} 0$ but as the receptor in $1 \mathrm{jsm}$ and $2 \mathrm{ibx}$, which further confirmed the different binding angles mentioned above. 


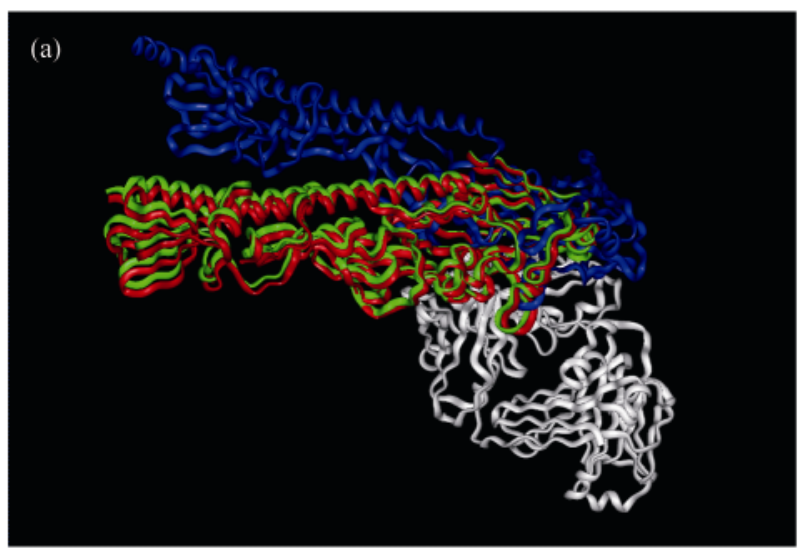

Figure 5 The binding pattern between $8 \mathrm{H} 5 \mathrm{Fab}$ and the 3 HAs. (a) White, $8 \mathrm{H} 5 \mathrm{Fab}$; red, 1jsm; green, 2ibx; blue, $2 \mathrm{fk} 0$. (b)-(d) The coloring region in surface was the binding region in HA. The binding sites in $8 \mathrm{H} 5$ were shown in ribbon. (b) Fab8H5-ljsm; (c) Fab8H5-2ibx; (d) Fab8H5-2fk0.

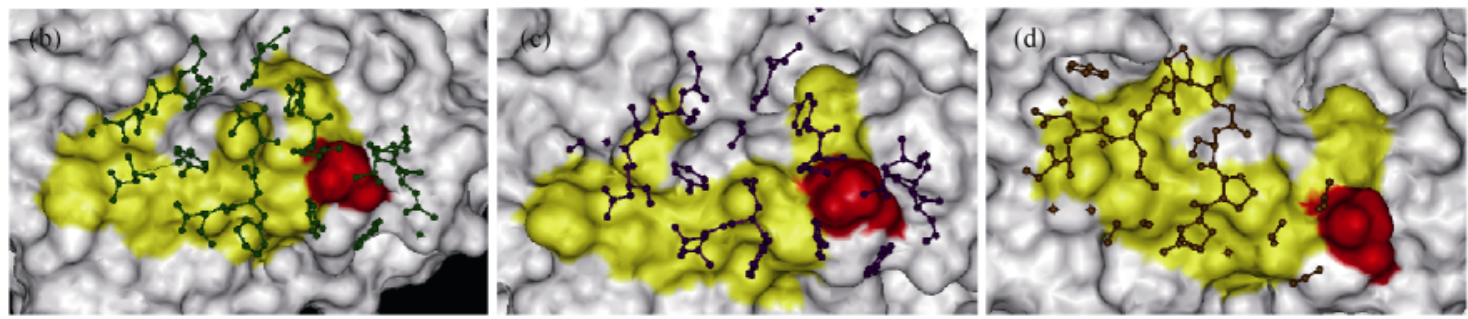

Table 5 SAS analysis of HA epitopes $\left(\AA^{2}\right)^{\text {a) }}$

\begin{tabular}{|c|c|c|c|c|c|c|c|c|}
\hline \multicolumn{3}{|c|}{$2 \mathrm{fk} 0$} & \multicolumn{3}{|c|}{$1 \mathrm{jsm}$} & \multicolumn{3}{|c|}{$2 \mathrm{ibx}$} \\
\hline Site & aa & $\triangle \mathrm{SAS}$ & Site & aa & $\triangle \mathrm{SAS}$ & Site & aa & $\Delta \mathrm{SAS}$ \\
\hline A57 & Lys & 78.361 & $\underline{\mathrm{A} 68}$ & Asp & 27.716 & $\underline{\mathbf{A 7 2}}$ & Asp & 18.55 \\
\hline A60 & Ile & 23.262 & A71 & Leu & 37.573 & A75 & Ile & 66.375 \\
\hline A62 & Arg & 70.997 & $\underline{\mathbf{A 7 2}}$ & $\underline{\text { Asn }}$ & 86.13 & $\underline{\mathrm{A} 76}$ & $\underline{\text { Asn }}$ & 65.577 \\
\hline A75 & Met & 8.945 & $\underline{\mathbf{A} 112}$ & Glu & 28.131 & $\underline{\mathbf{A 1 1 6}}$ & Glu & 18.239 \\
\hline$\underline{\mathbf{A} 77}$ & $\underline{\text { Asp }}$ & 40.472 & $\underline{\mathbf{A 1 1 3}}$ & $\underline{\text { Lys }}$ & 30.212 & $\underline{\mathbf{A} 117}$ & $\underline{\text { Lys }}$ & 28.056 \\
\hline A78 & Glu & 26.316 & $\underline{\mathbf{A 1 1 4}}$ & $\underline{\text { Ile }}$ & 34.115 & $\underline{\mathbf{A} 118}$ & Ile & 34.576 \\
\hline A79 & Phe & 143.975 & A115 & Arg & 133.757 & A119 & Gln & 84.019 \\
\hline$\underline{\mathrm{A} 81}$ & $\underline{\text { Asn }}$ & 126.082 & A116 & Ile & 7.654 & $\underline{\mathbf{A} 122}$ & $\underline{\text { Pro }}$ & 35.802 \\
\hline A 82 & Val & 51.191 & $\underline{\mathbf{A} 118}$ & $\underline{\text { Pro }}$ & 48.655 & A123 & Lys & 52.91 \\
\hline A 82 & Pro & 69.096 & A119 & Arg & 28.444 & A124 & $\underline{\text { Ser }}$ & 104.211 \\
\hline A 83 & Glu & 10.926 & $\underline{\mathbf{A 1 2 0}}$ & $\underline{\text { Ser }}$ & 109.74 & A 125 & Ser & 41.273 \\
\hline A117 & His & 18.864 & A121 & Ser & 30.395 & A126 & Trp & 9.254 \\
\hline A118 & Phe & 7.108 & A123 & Ser & 68.298 & A 127 & Ser & 69.711 \\
\hline$\underline{\text { A119 }}$ & Glu & 56.822 & A 124 & Asn & 23.362 & A 130 & Glu & 17.771 \\
\hline$\underline{\mathbf{A 1 2 0}}$ & $\underline{\text { Lys }}$ & 49.1 & A126 & Asp & 8.974 & A135 & Val & 11.537 \\
\hline$\underline{A 121}$ & Ile & 31.501 & A 127 & Ala & 17.753 & A141 & $\underline{\text { Tyr }}$ & 54.689 \\
\hline A122 & Gln & 73.071 & A131 & Val & 8.408 & A142 & Gln & 28.543 \\
\hline A123 & Ile & 8.25 & $\mathbf{A 1 3 7}$ & $\underline{\text { Tyr }}$ & 47.138 & A 144 & Lys & 43.149 \\
\hline$\underline{\mathbf{A 1 2 5}}$ & $\underline{\text { Pro }}$ & 12.846 & A 140 & Arg & 37.735 & A146 & Ser & 13.358 \\
\hline A125 & $\underline{\text { Ser }}$ & 12.562 & A142 & Ser & 15.934 & A149 & Arg & 60.859 \\
\hline$\underline{\text { A141 }}$ & Tyr & 33.14 & A145 & Arg & 66.956 & A 157 & Lys & 69.367 \\
\hline A142 & Gln & 45.85 & A 153 & Lys & 50.086 & A158 & Asn & 10.379 \\
\hline$\underline{\mathrm{A} 256}$ & $\underline{\text { Tyr }}$ & 37.194 & A 154 & Asn & 64.563 & A 255 & Glu & 11.505 \\
\hline A 273 & Glu & 73.261 & A164 & Tyr & 9.15 & A256 & $\underline{\text { Tyr }}$ & 65.716 \\
\hline A 274 & Tyr & 56.55 & A251 & Glu & 7.993 & & & \\
\hline A276 & Asn & 77.474 & $\underline{\mathbf{A} 252}$ & $\underline{\text { Tyr }}$ & 52.789 & & & \\
\hline
\end{tabular}

a) The predicted sites shown in bold underlined style were the 9 common epitope amino acid residues from three docking results. 


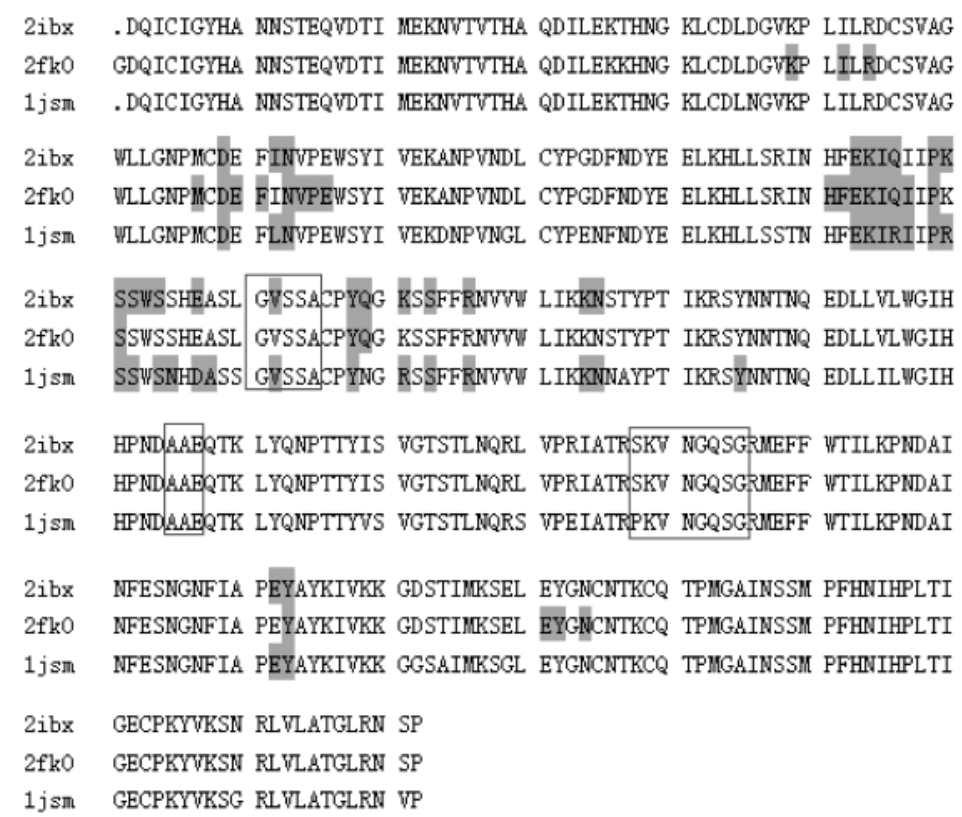

Figure 6 Sequence alignment to analyze the common epitope. Box, The reported receptor binding sites. Gray style means the 8H5 epitope amino acid residues predicted by SAS analysis as shown in Table 5 .

Table 6 Natural mutation ratio of the common epitope amino acids

\begin{tabular}{|c|c|c|c|c|c|c|c|c|c|}
\hline & \multicolumn{9}{|c|}{ Epitope amino acids in $8 \mathrm{H} 5$} \\
\hline & $\mathrm{Asp}^{68}$ & $\mathrm{Asn}^{72}$ & Glu $^{112}$ & Lys $^{113}$ & $\mathrm{Ile}^{114}$ & Pro $^{118}$ & $\operatorname{Ser}^{120}$ & $\operatorname{Tyr}^{137}$ & $\operatorname{Tyr}^{252}$ \\
\hline Cys & - & - & - & - & - & - & $0.1 \%$ & - & - \\
\hline Asp & $99.9 \%$ & - & $0.1 \%$ & - & - & - & $0.6 \%$ & - & - \\
\hline Glu & - & - & $99.6 \%$ & - & - & - & - & - & - \\
\hline Phe & - & - & - & - & - & - & - & $0.1 \%$ & - \\
\hline Gly & $0.1 \%$ & - & $0.2 \%$ & - & - & - & $0.2 \%$ & - & - \\
\hline Ile & - & - & - & - & $99.3 \%$ & - & - & - & - \\
\hline Lys & - & $0.3 \%$ & $0.1 \%$ & $99.9 \%$ & - & - & - & - & - \\
\hline Leu & - & - & - & - & $0.5 \%$ & - & - & - & - \\
\hline Met & - & - & - & - & - & - & - & - & - \\
\hline Asn & - & $99.5 \%$ & - & - & - & - & $3.2 \%$ & - & $12.6 \%$ \\
\hline Pro & - & - & - & - & - & $99.8 \%$ & - & - & - \\
\hline Gln & - & - & - & - & - & - & - & - & - \\
\hline Ser & - & - & - & - & - & $0.2 \%$ & $95.9 \%$ & $0.1 \%$ & - \\
\hline Thr & - & $0.1 \%$ & - & - & - & - & - & - & - \\
\hline Val & - & - & - & - & $0.3 \%$ & - & - & - & - \\
\hline Tyr & - & - & - & - & - & - & - & $99.1 \%$ & $87.2 \%$ \\
\hline
\end{tabular}

\section{Discussion}

The HA glycoprotein is present in the viral membrane as a single polypeptide (HA0), which must be cleaved by the hosts trypsin-like proteases to produce two peptides (HA1 and HA2) to make the virus infectious. HA may not only mediate the virus attaching to and entering into the host cell by binding to the sialic acid receptors on the cell surface, but also works as the main antigen that induces the humoral response against the virus ${ }^{[17]}$. There- fore, the HA is an important target for both drug and vaccine development. The epitopes of HA can be used to accurately monitor immune responses and to tease out which influenza responses are specific for a given virus isolate or subtype. They could also be used in detecting and monitoring infections serving to project potential cross-reactive immunity and efficacy against new isolates by existing vaccines and diagnostics. One of the shortcomings of the currently available influenza vaccines is the induction of a strain-specific immunity, 
Table 7 The hydrogen bond in the HA-8H5Fab complex

\begin{tabular}{|c|c|c|c|}
\hline H receptor & $\mathrm{H}$ donor & Distance & Angle \\
\hline 8H5Fab:H25:HG1 & 1jsm:A68:OD2 ${ }^{\text {b) }}$ & 2.36 & 139.11 \\
\hline 1jsm:A72:HD21 & 8H5Fab:H76:OD1 & 2.28 & 156.55 \\
\hline 1jsm:A72:HD22 & 8H5Fab:H27:O & 1.78 & 129.68 \\
\hline 8H5Fab:H31:HD22 & 1jsm:A112:OE2 & 1.93 & 139.49 \\
\hline 8H5Fab:H28:HG1 & 1jsm:A113:O ${ }^{\text {c) }}$ & 1.78 & 130.03 \\
\hline 1jsm:A115:HN & 8H5Fab:H32:OH & 1.81 & 124.02 \\
\hline 1jsm:A115:HN & 8H5Fab:H97:OD1 & 2.06 & 152.29 \\
\hline 1jsm:A115:HH22 & 8H5Fab:H94:OD1 & 1.70 & 153.24 \\
\hline 8H5Fab:L55:HN & 1jsm:A125:O & 1.87 & 138.59 \\
\hline 8H5Fab:L55:HN & 2ibx:A129:O & 2.33 & 120.20 \\
\hline 2ibx:A157:HZ1 & 8H5Fab:L55:O & 1.81 & 126.66 \\
\hline 2ibx:A157:HZ1 & $8 \mathrm{H} 5 \mathrm{Fab}: \mathrm{L} 56: \mathrm{N}$ & 2.45 & 126.52 \\
\hline 2ibx:A76:HD22 & 8H5Fab:H28:N & 2.41 & 135.04 \\
\hline$\overline{8 \mathrm{H} 5 \mathrm{Fab}: \mathrm{H} 28: \mathrm{HG} 1}$ & 2ibx:A117:O & 1.81 & 123.53 \\
\hline 8H5Fab:H31:HD22 & 2ibx:A116:OE2 & 1.91 & 142.38 \\
\hline 8H5Fab:H97:HN & 2ibx:A119:OE1 & 2.11 & 150.73 \\
\hline 2ibx:A119:HN & 8H5Fab:H97:OD1 & 1.89 & 136.25 \\
\hline 2fk0:A117:NE2 & 8H5Fab:L53:OD1 & 2.57 & $\mathrm{NA}^{\mathrm{a})}$ \\
\hline 2fk0:A120:HN & 8H5Fab:L54:O & 2.37 & 136.82 \\
\hline 2fk0:A256:HH & 8H5Fab:L57:O & 1.94 & 147.24 \\
\hline 2fk0:A62:HH21 & 8H5Fab:H26:O & 1.68 & 127.18 \\
\hline 8H5Fab:H28:HG1 & 2fk0:A273:OE1 & 2.25 & 143.37 \\
\hline 8H5Fab:H28:HG1 & 2fk0:A273:OE2 & 1.89 & 124.43 \\
\hline 2fk0:A57:HZ2 & 8H5Fab:H98:O & 1.76 & 125.63 \\
\hline 2fk0:A276:HD21 & 8H5Fab:H94:OH & 2.03 & 138.56 \\
\hline 2fk0:A79:HN & 8H5Fab:H102:OH & 2.06 & 151.32 \\
\hline
\end{tabular}

a) NA, No data was detected. b) Underlining style, the atoms of predicted 8H5 amino acid residues are contributing to from hydrogen bold in HA-8H5Fab complex. c) Underlining bold style, the atoms of predicted common $8 \mathrm{H} 5$ amino acid residues are contributing to from hydrogen bold in HA-8H5Fab complex.

which requires new vaccines to be produced each year for each different strain. For this reason, if conserved common epitope can be defined, different immunization regimens and vaccine candidates could be evaluated due to their capacity to induce immune responses to those specific conserved determinants ${ }^{[18]}$.

Damien Flury group reported that the epitopes of hemagglutinin were recognized by two distinct antibodies $^{[14]}$. But what they have done was focused on H3 that caused pandemics in 1968. However, the damage caused by avian influenza viruses since 1997 mostly derives from $\mathrm{H} 5$, and currently there is no X-ray resolved structure in the seeking for neutralizing epitope of H5. In this study, based on the experiment screening of the common monoclonal antibody $8 \mathrm{H} 5$, we constructed the structure using the molecule modeling and analyzed the binding pattern between the antibody and the three HAs so as to shed light on the conserved neutralizing epitope in HA.

The function of the antibody was mainly derived from the variable domains, and a lot of work was focused on the single-chain $\mathrm{FV}$ antibody ${ }^{[19,20]}$. In order to better screen results from the Zdock and Rdock poses which were carried out to find the interacting characteristic of the antibody and the antigen, we modeled the whole Fab structure. By analyzing the primary sequences of the variable domains, the framework and the canonical structure were determined for molecule modeling (Table 1). Based on the high consensus, the structure of framework could be predicted with high accuracy. And the CDRs could be predicted with the most accurate canonical structure method whose class was based on the length of the CDR and the key residue. Each canonical structure class possesses its individual conformation. This is the basic principle for the CDRs conformation determination. The structure modeled by canonical structure method was in the reasonable conformation after being tested by energy analysis, SAS analysis, Ramanchandran plot, profile-3D and superimposing with the X-ray resolved structures. As one of the best programs, Zdock was employed in the $8 \mathrm{H} 5 \mathrm{Fab}$ and HAs 
study. To verify the accuracy, the HA and antibody were separated from the H3 complex mentioned above and then subjected to the Zdock and Rdock analysis. The result was almost the same as that of X-ray resolved complex, indicating that the Zdock and Rdock program could perform very well in the antibody and antigen docking.

From the complexes of $8 \mathrm{H} 5 \mathrm{Fab}$ and HAs, it is easy to find out that the binding pattern of $8 \mathrm{H} 5 \mathrm{Fab}-1 \mathrm{jsm}$ and $8 \mathrm{H} 5 \mathrm{Fab}-2 \mathrm{ibx}$ was almost the same although their hosts were different. This result also indicated that the epitope against $8 \mathrm{H} 5$ was unrelated to the host, which implied that $8 \mathrm{H} 5$ can be extensively used in the diagnostic of HA antigens from different $\mathrm{H} 5$ influenza viruses. However, the binding pattern of $8 \mathrm{H} 5 \mathrm{Fab}-2 \mathrm{fk} 0$ was quite different from the other two. The $2 \mathrm{fk} 0$ structure was more similar to H1 HAs than $\mathrm{H} 5 \mathrm{HA}$, and from the experiment, we found that $8 \mathrm{H} 5$ cannot react with $\mathrm{HA}$ form $\mathrm{H} 1$ subtype. This result showed that there was HA subtype binding preference for $8 \mathrm{H} 5$, which could provide crucial information for the research of HA subtype transition from H5 to H1. Taking consideration of the $\triangle \mathrm{SAS}$ at the interface of the complexes and the homologous sequence alignment, the epitope against $8 \mathrm{H} 5$ was: (the residue No.

1 Luo H F, Chen Y X, Chen Z M, et al. Characterization of a broadspectrum neutralization monoclonal antibody against haemagglutinin of H5 subtype avian influenza virus. Chin J Virol (in Chinese), 2007, 23(2): $85-90$

2 Bruccoleri R E, Haber E, Novotny J. Structure of antibody hypervariable loops reproduced by a conformational search algorithm. Nature, 1988, 335: 564-568

3 Chothia C, Lesk A M, Tramontano A, et al. Conformations of immunoglobulin hypervariable regions. Nature, 1989, 342: 877-883

4 Chothia C, Lesk A M. Canonical structures for the hypervariable loops of immunoglobulins. J Mol Biol, 1987, 196: 901

5 Veronica M, Arthur M L, Anna T. Antibody modeling: Implications for engineering and design. Methods, 2000, 20: 267-279

6 Stevens J, Blixt O, Tumpey T M, et al. Structure and receptor specificity of the hemagglutinin from an $\mathrm{H} 5 \mathrm{~N} 1$ influenza virus. Science, 2006, 312: 404-410

7 Yamada S, Suzuki Y, Suzuki T, et al. Haemagglutinin mutations responsible for the binding of H5N1 influenza A viruses to Human-type receptors. Nature, 2006, 444: 378-382

8 Ha Y, Stevens D J, Skehel J J, et al. H5 avian and H9 swine influenza virus haemagglutinin structures: Possible origin of influenza subtype. EMBO J, 2002, 21: 865-875

9 Lipman D J, Pearson W R. Rapid and sensitive protein similarity searches. Science, 1985, 227: 1435-1441

10 Pearson W R. Rapid and sensitive sequence comparison with FASTP and FASTA. Methods Enzymol, 1990, 183: 63-98

11 Chothia C, Novotny J, Bruccoleri R, et al. Domain association in immunoglobulin molecules. The packing of variable domains. J Mol in 1 jsm) $\mathrm{Asp}^{68}, \mathrm{Asn}^{72}, \mathrm{Glu}^{112}, \mathrm{Lys}^{113}, \mathrm{Ile}^{114}, \operatorname{Pro}^{118}, \mathrm{Ser}^{120}$, $\mathrm{Tyr}^{137}, \mathrm{Tyr}^{252}$. The epitope was located in three small regions near the receptor binding sub-domain, and all of epitope amino acids were in the random coil with exception of about 4 residues in beta sheet. The one ranging from $\mathrm{Glu}^{112}$ to $\mathrm{Ser}^{121}$ in $1 \mathrm{jsm}$ was the largest one that possessed 10 residues. Interestingly, the two epitopes regions from $\mathrm{Glu}^{112}$ to $\mathrm{Ser}^{121}$ and from $\mathrm{Tyr}^{137}$ to $\mathrm{Lys}^{140}$ in $1 \mathrm{jsm}$ had been confirmed as the favorite antigen binding sites reported by James Stevens group in $2006^{[6]}$. Besides, the natural mutation ratio also showed that the conservation ratio of the predicted common neutralizing epitope was very high. Because the hydrogen bond played an important role in the protein-protein interaction, Lys ${ }^{113}$ might be the key amino acid in the antibody and antigen binding, which might offer crucial information for the anti-virus drug design. However, more gene mutation, epitope analysis ${ }^{[21]}$ and structure resolved experiments should be performed to verify the structure and binding pattern.

The authors are grateful to Dr. Han Jinhua, Dr. Kong Yong at National University of Singapore and Dr. Lin Tianwei at Scripps Research Center for assistances.

Biol, 1985, 186: 651-663

12 Chen R, Weng Z. A novel shape complementarity scoring function for protein-protein docking. Proteins, 2003, 51: 397-408

13 Li L, Chen R, Weng Z. RDOCK: Refinement of rigid-body protein docking predictions. Proteins, 2003, 53: 693-707

14 Fleury D, Daniels R S, Skehel J J, et al. Structural Evidence for recognition of a single epitope by two distinct antibodies. Proteins, 2000, 40: $572-578$

15 Lee B, Richards F M. The interpretation of protein structures: Estimation of static accessibility. J Mol Biol, 1971, 55: 379-400

16 Connolly M L. Solvent-accessible surfaces of proteins and nucleic acids. Science, 1983, 221: 709-713

17 Thomas J K, Noppenberger J. Avian influenza: A review. Am J Health Syst Pharm, 2007, 64: 149-165

18 Bui H H, Peters B, Assarsson E, et al. Ab and T cell epitopes of influenza A virus, knowledge and opportunities. Proc Natl Acad Sci USA, 2007, 104: 246-251

19 Morioka H, Miura H, Kobayashi H, et al. Antibodies specific for (6-4) DNA photoproducts: Cloning, antibody modeling and construction of a single-Chain Fv derivative. Biochim Biophys Acta, 1998, 1385: 17-32

20 Guo C Z, Wu J H, Wang Y X, et al. Molecular simulation of a single-chain antibody against $\mathrm{AChE}$ to explore molecular basis of inhibitory effect of $3 \mathrm{~F} 3 \mathrm{McAb}$ to enezyme activity. Acta Pharmacol Sin, 2003, 24: 460-466

21 Li G, Tao S, Wang X J. Sequence and epitope analysis of surface proteins of avian influenza $\mathrm{H} 5 \mathrm{~N} 1$ viruses from Asian patients. Chin Sci Bull, 2006, 51: 2472-2481 